\title{
The Derivations of Temporal Logic Formulas $^{1}$
}

\author{
Mariusz Giero ${ }^{2}$ \\ Department of Logic, Informatics and Philosophy of Science \\ University of Białystok \\ Plac Uniwersytecki 1, 15-420 Białystok, Poland
}

\begin{abstract}
Summary. This is a preliminary article to prove the completeness theorem of an extension of basic propositional temporal logic. We base it on the proof of completeness for basic propositional temporal logic given in [12]. We introduce $n$-ary connectives and prove their properties. We derive temporal logic formulas.
\end{abstract}

MML identifier: LTLAXI02, version: $\underline{7.14 .01 \quad 4.183 .1153}$

The papers [14], [3], [1], [16], [6], [17], [8], [2], [7], [13], [4], [5], [11], [10], [15], and [9] provide the terminology and notation for this paper.

\section{Preliminaries}

For simplicity, we adopt the following rules: $A, B, p, q, r, s$ are elements of the LTLB-WFF, $i, k, n$ are elements of $\mathbb{N}, X$ is a subset of the LTLB-WFF, $f$, $f_{1}$ are finite sequences of elements of the LTLB-WFF, and $g$ is a function from the LTLB-WFF into Boolean.

Let $f$ be a finite sequence and let $x$ be an empty set. One can check that $f(x)$ is empty.

We now state three propositions:

\footnotetext{
${ }^{1}$ This work has been supported by the Polish Ministry of Science and Higher Education project "Managing a Large Repository of Computer-verified Mathematical Knowledge" (N N519 385136).

${ }^{2}$ I would like to thank Prof. Andrzej Trybulec, Dr. Artur Korniłowicz, Dr. Adam Naumowicz and Karol Pąk for their help in preparation of the article.

(C) 2012 University of Białystok CC-BY-SA License ver. 3.0 or later ISSN 1426-2630(p), 1898-9934(e)
} 
(1) For every finite sequence $f$ such that $\operatorname{len} f>0$ and $n>0$ holds $\operatorname{len}(f\lceil n)>0$.

(2) For every finite sequence $f$ such that len $f=0$ holds $f_{\lfloor n}=f$.

(3) For all finite sequences $f, g$ such that $\operatorname{rng} f=\operatorname{rng} g$ holds len $f=0$ iff len $g=0$.

Let us consider $A, B$. The functor $\mathrm{UN}(A, B)$ yields an element of the LTLB$\mathrm{WFF}$ and is defined by:

(Def. 1) $\quad \mathrm{UN}(A, B)=B \vee(A \& \&(A \mathcal{U} B))$.

One can prove the following proposition

(4) $\operatorname{VAL}_{g}\left(T_{t}\right)=1$.

Next we state the proposition

(5) $\operatorname{VAL}_{g}(p \vee q)=\operatorname{VAL}_{g}(p) \vee \operatorname{VAL}_{g}(q)$.

\section{2. $n$-Argument Connectives And their Properties}

Let us consider $f$. The functor conjunction $f$ yielding a finite sequence of elements of the LTLB-WFF is defined as follows:

(Def. 2)(i) len conjunction $f=\operatorname{len} f$ and (conjunction $f)(1)=f(1)$ and for every $i$ such that $1 \leq i<\operatorname{len} f$ holds $($ conjunction $f)(i+1)=$ (conjunction $f)_{i} \& \& f_{i+1}$ if len $f>0$,

(ii) conjunction $f=\left\langle\top_{t}\right\rangle$, otherwise.

Let us consider $f, A$. The functor implication $(f, A)$ yielding a finite sequence of elements of the LTLB-WFF is defined as follows:

(Def. 3)(i) len implication $(f, A)=\operatorname{len} f$ and $(\operatorname{implication}(f, A))(1)=\mathcal{G}\left(f_{1}\right) \Rightarrow A$ and for every $i$ such that $1 \leq i<\operatorname{len} f$ holds (implication $(f, A))(i+1)=$ $\mathcal{G}\left(f_{i+1}\right) \Rightarrow(\text { implication }(f, A))_{i}$ if len $f>0$,

(ii) $\operatorname{implication}(f, A)=\varepsilon_{\text {(the LTLB-WFF) }}$, otherwise.

Let us consider $f$. The functor negation $f$ yields a finite sequence of elements of the LTLB-WFF and is defined by:

(Def. 4) len negation $f=\operatorname{len} f$ and for every $i$ such that $1 \leq i \leq \operatorname{len} f$ holds (negation $f)(i)=\neg\left(f_{i}\right)$.

Let us consider $f$. The functor next $f$ yields a finite sequence of elements of the LTLB-WFF and is defined by:

(Def. 5) len next $f=\operatorname{len} f$ and for every $i$ such that $1 \leq i \leq \operatorname{len} f$ holds $($ next $f)(i)=\mathcal{X}\left(f_{i}\right)$.

We now state a number of propositions:

(6) If len $f>0$, then (conjunction $f)_{1}=f_{1}$.

(7) For every natural number $i$ such that $1 \leq i<\operatorname{len} f$ holds $(\text { conjunction } f)_{i+1}=(\operatorname{conjunction} f)_{i} \& \& f_{i+1}$. 
(8) For every natural number $i$ such that $i \in \operatorname{dom} f$ holds $(\text { negation } f)_{i}=$ $\neg\left(f_{i}\right)$.

(9) For every natural number $i$ such that $i \in \operatorname{dom} f$ holds (next $f)_{i}=\mathcal{X}\left(f_{i}\right)$.

(10) $\left(\operatorname{conjunction}\left(\varepsilon_{(\text {the LTLB-WFF })}\right)\right)_{\text {len conjunction }\left(\varepsilon_{(\text {the LTLB-WFF })}\right)}=\top_{t}$.

(11) (conjunction $\langle A\rangle)_{\text {len conjunction }\langle A\rangle}=A$.

(12) For every $k$ such that $n \leq k$ holds $($ conjunction $f)(n)=$ (conjunction $(f\lceil k))(n)$.

(13) For every $k$ such that $n \leq k$ and $1 \leq n \leq \operatorname{len} f$ holds $(\operatorname{conjunction} f)_{n}=$ (conjunction $(f\lceil k))_{n}$.

(14) negation $\langle A\rangle=\langle\neg A\rangle$.

(15) $\operatorname{negation}\left(f^{\frown}\langle A\rangle\right)=(\text { negation } f)^{\frown}\langle\neg A\rangle$.

(16) $\operatorname{negation}\left(f \frown f_{1}\right)=(\text { negation } f)^{\frown}$ negation $f_{1}$.

(17) $\operatorname{VAL}_{g}\left(\left(\text { conjunction }\left(f \frown f_{1}\right)\right)_{\text {len conjunction }\left(f \frown f_{1}\right)}\right)=$ $\operatorname{VAL}_{g}\left((\text { conjunction } f)_{\text {len conjunction } f}\right) \wedge \operatorname{VAL}_{g}\left(\left(\right.\right.$ conjunction $\left.f_{1}\right)$ len conjunction $\left.f_{1}\right)$.

(18) If $n \in \operatorname{dom} f$, then $\operatorname{VAL}_{g}\left((\operatorname{conjunction} f)_{\text {len conjunction } f}\right)=$ $\operatorname{VAL}_{g}\left(\left(\text { conjunction }\left(f \uparrow\left(n-{ }^{\prime} 1\right)\right)\right)_{\text {len conjunction }\left(f \uparrow\left(n-^{\prime} 1\right)\right)}\right) \wedge \operatorname{VAL}_{g}\left(f_{n}\right) \wedge$ $\operatorname{VAL}_{g}\left(\left(\text { conjunction }\left(f_{\lfloor n}\right)\right)_{\text {len conjunction }\left(f_{\lfloor n}\right)}\right)$.

(19) $\operatorname{VAL}_{g}\left((\operatorname{conjunction} f)_{\text {len conjunction } f}\right)=1$ iff for every natural number $i$ such that $i \in \operatorname{dom} f$ holds $\operatorname{VAL}_{g}\left(f_{i}\right)=1$.

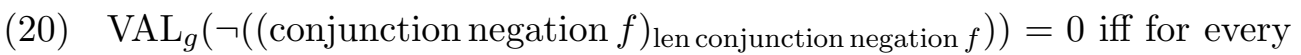
natural number $i$ such that $i \in \operatorname{dom} f$ holds $\operatorname{VAL}_{g}\left(f_{i}\right)=0$.

(21) If $\operatorname{rng} f=\operatorname{rng} f_{1}$, then $\operatorname{VAL}_{g}\left((\text { conjunction } f)_{\text {len conjunction } f}\right)=$ $\operatorname{VAL}_{g}\left(\left(\right.\right.$ conjunction $\left.f_{1}\right)$ len conjunction $\left.f_{1}\right)$.

\section{Classical Tautologies of Temporal Language}

Next we state a number of propositions:

(22) $\quad p \Rightarrow \top_{t}$ is tautologically valid.

(23) $\neg \top_{t} \Rightarrow p$ is tautologically valid.

(24) $p \Rightarrow p$ is tautologically valid.

(25) $\neg \neg p \Rightarrow p$ is tautologically valid.

(26) $\quad p \Rightarrow \neg \neg p$ is tautologically valid.

(27) $\quad p \& \& q \Rightarrow p$ is tautologically valid.

(28) $\quad p \& \& q \Rightarrow q$ is tautologically valid.

(29) For every natural number $k$ such that $k \in \operatorname{dom} f$ holds $f_{k} \Rightarrow$ $\neg\left((\text { conjunction negation } f)_{\text {len conjunction negation } f)}\right)$ is tautologically valid.

(30) If $\operatorname{rng} f \subseteq \operatorname{rng} f_{1}$, then $\neg\left((\text { conjunction negation } f)_{\text {len conjunction negation } f) \Rightarrow}\right)$ $\neg\left(\left(\right.\right.$ conjunction negation $\left.f_{1}\right)$ len conjunction negation $\left.f_{1}\right)$ is tautologically valid. 
(31) $\neg(p \Rightarrow q) \Rightarrow p$ is tautologically valid.

(32) $\neg(p \Rightarrow q) \Rightarrow \neg q$ is tautologically valid.

(33) $p \Rightarrow(q \Rightarrow p)$ is tautologically valid.

(34) $\quad p \Rightarrow(q \Rightarrow(p \Rightarrow q))$ is tautologically valid.

(35) $\neg(p \& \& q) \Rightarrow \neg p \vee \neg q$ is tautologically valid.

(36) $\neg(p \vee q) \Rightarrow \neg p \& \& \neg q$ is tautologically valid.

(37) $\neg(p \& \& q) \Rightarrow(p \Rightarrow \neg q)$ is tautologically valid.

(38) $\neg\left(\top_{t} \& \& \neg A\right) \Rightarrow A$ is tautologically valid.

(39) $\neg(s \& \& q) \Rightarrow((p \Rightarrow q) \Rightarrow(p \Rightarrow \neg s))$ is tautologically valid.

(40) $(p \Rightarrow r) \Rightarrow((p \Rightarrow s) \Rightarrow(p \Rightarrow r \& \& s))$ is tautologically valid.

(41) $\neg(p \& \& s) \Rightarrow \neg(r \& \& s \& \&(p \& \& q))$ is tautologically valid.

(42) $\neg(p \& \& s) \Rightarrow \neg(p \& \& q \& \&(r \& \& s))$ is tautologically valid.

(43) $\quad(p \Rightarrow q \& \& \neg q) \Rightarrow \neg p$ is tautologically valid.

(44) $\quad(q \Rightarrow p \& \& r) \Rightarrow((p \Rightarrow s) \Rightarrow(q \Rightarrow s \& \& r))$ is tautologically valid.

(45) $(p \Rightarrow q) \Rightarrow((r \Rightarrow s) \Rightarrow(p \& \& r \Rightarrow q \& \& s))$ is tautologically valid.

(46) $\quad(p \Rightarrow q) \Rightarrow((p \Rightarrow r) \Rightarrow((r \Rightarrow p) \Rightarrow(r \Rightarrow q)))$ is tautologically valid.

(47) $(p \Rightarrow q) \Rightarrow((p \Rightarrow \neg r) \Rightarrow(p \Rightarrow \neg(q \Rightarrow r)))$ is tautologically valid.

(48) $\quad(p \Rightarrow q \vee r) \Rightarrow((r \Rightarrow s) \Rightarrow(p \Rightarrow q \vee s))$ is tautologically valid.

(49) $\quad(p \Rightarrow r) \Rightarrow((q \Rightarrow r) \Rightarrow(p \vee q \Rightarrow r))$ is tautologically valid.

(50) $\quad(r \Rightarrow \mathrm{UN}(p, q)) \Rightarrow((r \Rightarrow \neg p \& \& \neg q) \Rightarrow \neg r)$ is tautologically valid.

(51) $\quad(r \Rightarrow \mathrm{UN}(p, q)) \Rightarrow((r \Rightarrow \neg q \& \& \neg(p \mathcal{U} q)) \Rightarrow \neg r)$ is tautologically valid.

\section{The Derivations of Temporal Logic Formulas within Classical LOGIC}

One can prove the following propositions:

(52) If $X \vdash p \Rightarrow q$ and $X \vdash p \Rightarrow r$, then $X \vdash p \Rightarrow q \& \& r$.

(53) If $X \vdash p \Rightarrow q$ and $X \vdash r \Rightarrow s$, then $X \vdash p \& \& r \Rightarrow q \& \& s$.

(54) If $X \vdash p \Rightarrow q$ and $X \vdash p \Rightarrow r$ and $X \vdash r \Rightarrow p$, then $X \vdash r \Rightarrow q$.

(55) If $X \vdash p \Rightarrow q \& \& \neg q$, then $X \vdash \neg p$.

(56) If for every natural number $i$ such that $i \in \operatorname{dom} f$ holds

$\emptyset_{\text {the LTLB-WFF }} \vdash p \Rightarrow f_{i}$, then

$\emptyset_{\text {the LTLB-WFF }} \vdash p \Rightarrow(\text { conjunction } f)_{\text {len conjunction } f}$.

(57) If for every natural number $i$ such that $i \in \operatorname{dom} f$ holds

$\emptyset_{\text {the LTLB-WFF }} \vdash f_{i} \Rightarrow p$, then

$\emptyset_{\text {the LTLB-WFF }} \vdash \neg\left((\text { conjunction negation } f)_{\text {len conjunction negation } f)} \Rightarrow p\right.$. 


\section{The Derivations of Temporal Logic Formulas}

Next we state several propositions:

(58) $X \vdash(\mathcal{X} p \Rightarrow \mathcal{X} q) \Rightarrow \mathcal{X}(p \Rightarrow q)$.

(59) $X \vdash \mathcal{X}(p \& \& q) \Rightarrow \mathcal{X} p \& \& \mathcal{X} q$.

(60) $\emptyset_{\text {the LTLB-WFF }} \vdash(\text { conjunction next } f)_{\text {len conjunction next } f} \Rightarrow$ $\mathcal{X}\left((\text { conjunction } f)_{\text {len conjunction } f)}\right.$.

(61) $X \vdash \mathcal{X} p \vee \mathcal{X} q \Rightarrow \mathcal{X}(p \vee q)$.

(62) $X \vdash \mathcal{X}(p \vee q) \Rightarrow \mathcal{X} p \vee \mathcal{X} q$

(63) $X \vdash \neg(A \mathcal{U} B) \Rightarrow \mathcal{X} \neg \mathrm{UN}(A, B)$.

\section{REFERENCES}

[1] Grzegorz Bancerek. Cardinal numbers. Formalized Mathematics, 1(2):377-382, 1990.

[2] Grzegorz Bancerek. The fundamental properties of natural numbers. Formalized Mathematics, 1(1):41-46, 1990.

[3] Grzegorz Bancerek. The ordinal numbers. Formalized Mathematics, 1(1):91-96, 1990.

[4] Grzegorz Bancerek and Krzysztof Hryniewiecki. Segments of natural numbers and finite sequences. Formalized Mathematics, 1(1):107-114, 1990.

[5] Czesław Byliński. Finite sequences and tuples of elements of a non-empty sets. Formalized Mathematics, 1(3):529-536, 1990.

[6] Czesław Byliński. Functions and their basic properties. Formalized Mathematics, 1(1):5565, 1990.

[7] Czesław Byliński. Functions from a set to a set. Formalized Mathematics, 1(1):153-164, 1990.

[8] Czesław Byliński. Partial functions. Formalized Mathematics, 1(2):357-367, 1990.

[9] Mariusz Giero. The axiomatization of propositional linear time temporal logic. Formalized Mathematics, 19(2):113-119, 2011, doi: 10.2478/v10037-011-0018-1.

[10] Adam Grabowski. Hilbert positive propositional calculus. Formalized Mathematics, 8(1):69-72, 1999.

[11] Jarosław Kotowicz. Functions and finite sequences of real numbers. Formalized Mathematics, 3(2):275-278, 1992.

[12] Fred Kröger and Stephan Merz. Temporal Logic and State Systems. Springer-Verlag, 2008.

[13] Andrzej Trybulec. Binary operations applied to functions. Formalized Mathematics, 1(2):329-334, 1990.

[14] Zinaida Trybulec. Properties of subsets. Formalized Mathematics, 1(1):67-71, 1990.

[15] Edmund Woronowicz. Many argument relations. Formalized Mathematics, 1(4):733-737, 1990.

[16] Edmund Woronowicz. Relations and their basic properties. Formalized Mathematics, 1(1):73-83, 1990.

[17] Edmund Woronowicz. Relations defined on sets. Formalized Mathematics, 1(1):181-186, 1990.

Received May 7, 2012 